\title{
Complicações crônicas decorrentes do Diabetes mellitus: uma revisão narrativa de
}

\section{literatura}

\author{
Chronic complications arising from Diabetes mellitus: a narrative literature review \\ Complicaciones crónicas derivadas de la Diabetes mellitus: una revisión narrativa de la literatura
}

Recebido: 14/10/2021 | Revisado: 23/10/2021 | Aceito: 24/10/2021 | Publicado: 27/10/2021

Bárbara Queiroz de Figueiredo

ORCID: https://orcid.org/0000-0003-1630-4597

Centro Universitário de Patos de Minas, Brasil

E-mail: barbarafigueiredo@unipam.edu.br

Ana Clara Viana Soares Brito

ORCID: https://orcid.org/0000-0001-5747-4280

Centro Universitário Uninovafapi, Brasil

E-mail: anaclaravsb@hotmail.com

Bianca Reis Carvalho Miranda

ORCID: https://orcid.org/0000-0001-9495-4646

Centro Universitário Barão de Mauá, Brasil E-mail: bibireiscm1@gmail.com

Isabelle Carvalho de Melo Lima

ORCID: https://orcid.org/0000-0002-1875-9117

Centro Universitário Uninovafapi, Brasil

E-mail: isabellemelo.carvalho@gmail.com

Isabelle Gomes de Sousa

ORCID: https://orcid.org/0000-0002-0067-019X

Centro Universitário Uninovafapi, Brasil

E-mail: isabellegmss@hotmail.com

Lunalva Gabrielli Veras Sousa

ORCID: https://orcid.org/0000-0001-5181-5371

Centro Universitário Uninovafapi, Brasil E-mail: veraslunalva@gmail.com

Stéfani do Vale

ORCID: https://orcid.org/0000-0002-5211-9834 Centro Universitário Atenas, Brasil

E-mail: stefani.vale@hotmail.com

Victoria Herrera de Souza

ORCID: https://orcid.org/0000-0002-9951-9640

Centro Universitário São Francisco de Barreiras, Brasil

E-mail: vi_victoriaherrera@hotmail.com

\begin{abstract}
Resumo
Introdução: o Diabetes mellitus (DM) é um conjunto de patologias que possuem, em comum, a hiperglicemia crônica. A doença e, principalmente, seu mau controle, podem causar o aumento da glicemia e as altas taxas podem levar a complicações no coração, nas artérias, nos olhos, nos rins e nos nervos, e, desse modo, o objetivo deste estudo é evidenciar as principais complicações crônicas decorrentes do diabetes mellitus. Objetivo: evidenciar as principais complicações crônicas decorrentes do diabetes mellitus. Metodologia: Trata-se de uma pesquisa descritiva do tipo revisão narrativa da literatura, que buscou responder quais são as evidências sobre as complicações a longo prazo do diabetes mellitus. A pesquisa foi realizada através do acesso online nas bases de dados National Library of Medicine (PubMed MEDLINE), Scientific Electronic Library Online (Scielo), Cochrane Database of Systematic Reviews (CDSR), Google Scholar, Biblioteca Virtual em Saúde (BVS) e EBSCO Information Services, no mês de outubro de 2021. Resultados e discussão: As complicações diabéticas crônicas são classificadas como microvasculares ou macrovasculares, e contribuem para mortalidade e perda de qualidade de vida de pacientes portadores de diabetes mellitus. Sob essa perspectiva, nota-se que a hiperglicemia desempenha um papel crítico na patogênese das complicações microvasculares, como retinopatia diabética, nefropatia incipiente e neuropatia, enquanto a aterosclerose contribui para a patogênese das complicações macrovasculares. Conclusão: é de suma importância, ao observar a relevância das medidas de estilo de vida, a adoção da prática dos exercícios físicos nas pessoas com DM, principalmente nos idosos, que se efetive o controle glicêmico dos portadores de DM2, e, assim, controlando as morbidades associadas e cronicidades.
\end{abstract}

Palavras-chave: Diabetes mellitus; Complicações; Nefropatia; Neuropatia; Retinopatia. 


\begin{abstract}
Introduction: Diabetes mellitus (DM) is a set of pathologies that have chronic hyperglycemia in common. The disease, and especially its poor control, can cause an increase in blood glucose and the high rates can lead to complications in the heart, arteries, eyes, kidneys and nerves, and, thus, the aim of this study is to demonstrate the main chronic complications resulting from diabetes mellitus. Objective: to highlight the main chronic complications resulting from diabetes mellitus. Methodology: This is a descriptive research of the narrative literature review type, which sought to answer what is the evidence on the long-term complications of diabetes mellitus. The search was carried out through online access in the National Library of Medicine (PubMed MEDLINE), Scientific Electronic Library Online (Scielo), Cochrane Database of Systematic Reviews (CDSR), Google Scholar, Virtual Health Library (BVS) and EBSCO databases Information Services, October 2021. Results and discussion: Chronic diabetic complications are classified as microvascular or macrovascular, and contribute to mortality and loss of quality of life in patients with diabetes mellitus. From this perspective, it is noted that hyperglycemia plays a critical role in the pathogenesis of microvascular complications, such as diabetic retinopathy, incipient nephropathy and neuropathy, while atherosclerosis contributes to the pathogenesis of macrovascular complications. Conclusion: it is of paramount importance, when observing the relevance of lifestyle measures, the adoption of the practice of physical exercise in people with DM, especially in the elderly, that the glycemic control of patients with DM2 be effective, and thus controlling associated morbidities and chronicities.
\end{abstract}

Keywords: Diabetes mellitus; Complications; Nephropathy; Neuropathy; Retinopathy.

\title{
Resumen
}

Introducción: la Diabetes mellitus (DM) es un conjunto de patologías que tienen en común la hiperglucemia crónica. La enfermedad, y sobre todo su mal control, puede provocar un aumento de la glucosa en sangre y las altas tasas pueden derivar en complicaciones en el corazón, arterias, ojos, riñones y nervios, por lo que el objetivo de este estudio es demostrar las principales Complicaciones crónicas resultantes de la diabetes mellitus. Objetivo: destacar las principales complicaciones crónicas derivadas de la diabetes mellitus.Metodología: Se trata de una investigación descriptiva del tipo revisión de la literatura narrativa, que buscó responder cuál es la evidencia sobre las complicaciones a largo plazo de la diabetes mellitus. La búsqueda se realizó a través del acceso online en la Biblioteca Nacional de Medicina (PubMed MEDLINE), Scientific Electronic Library Online (Scielo), Cochrane Database of Systematic Reviews (CDSR), Google Scholar, Virtual Health Library (BVS) y bases de datos EBSCO Information Services, Octubre de 2021. Resultados y discusión: Las complicaciones diabéticas crónicas se clasifican en microvasculares o macrovasculares y contribuyen a la mortalidad y pérdida de calidad de vida en pacientes con diabetes mellitus. Desde esta perspectiva, se observa que la hiperglucemia juega un papel crítico en la patogenia de las complicaciones microvasculares, como la retinopatía diabética, la nefropatía incipiente y la neuropatía, mientras que la aterosclerosis contribuye a la patogénesis de las complicaciones macrovasculares. Conclusión: es de suma importancia, al observar la relevancia de las medidas de estilo de vida, la adopción de la práctica de ejercicio físico en personas con DM, especialmente en los ancianos, que el control glucémico de los pacientes con DM2 sea efectivo, y así controlar las morbilidades asociadas y cronicidades.

Palabras clave: Diabetes mellitus; Complicaciones; Nefropatía; Neuropatía; Retinopatía.

\section{Introdução}

O Diabetes mellitus (DM) é um conjunto de patologias que possuem, em comum, a hiperglicemia crônica. No DM do tipo 1 (DM1) ocorre uma deficiência total ou quase total da produção de insulina pelas células $\beta$ das ilhotas pancreáticas (ilhotas de Langerhans). Na maioria dos casos, este déficit de secreção de insulina deriva da destruição das células $ß$ por mecanismos de autoimunidade, e pequena parte desta falência de produção é considerada idiopática. Já o DM do tipo 2 (DM2) é uma patologia de herança poligênica desencadeada, na maioria das vezes, por fatores ambientais como sedentarismo e hábitos alimentares pouco saudáveis. Isto culmina com aumento do tecido adiposo corporal (especialmente visceral) e produção de citocinas inflamatórias por este tecido. A ação dessas citocinas em todo o corpo determina resistência à ação da insulina nos tecidos (Hang et al., 2019).

No que se refere ao diabetes mellitus do tipo 2, (DM2), que resulta, em graus variáveis, da resistência tecidual à insulina e deficiência relativa na secreção do hormônio pelas células $\beta$ pancreática, apesar da forte herança familiar poligênica ainda não esclarecida, o fator ambiental é muito relevante para o desenvolvimento desta patologia, haja vista que os hábitos dietéticos e o sedentarismo são os principais contribuintes para a obesidade, que é o principal fator de risco para o desenvolvimento do DM2. Na maioria dos casos, o acúmulo de gordura, especialmente no tecido adiposo visceral, é associado 
ao risco de DM2. Neste tecido, quando há hipertrofia, são produzidas citocinas pró-inflamatórias, o que gera uma resistência à insulina, que está envolvida na gênese do DM2 (Freitas et al., 2020). Em um estado anormal ou resistente à insulina, há uma perda da secreção de insulina parcial em uma resposta a uma carga de glicose, resultando em hiperglicemia pós-prandial. Posteriormente, um exagerado a resposta à insulina de segunda fase causa hiperinsulinemia crônica (Hang, et al. 2019). Ou seja, a resistência à insulina se manifesta como uma redução na capacidade da insulina de ativar o sinal de insulina celularcascata e, consequentemente, estimular a insulina em processos celulares calculados. A fisiopatologia do diabetes mellitus de tipo 2 é impulsionada principalmente pela indução da resistência à insulina muscular, hepática e do tecido adiposo (Prasad et al., 2014).

Nota-se que a DM2, principalmente, possui etiologia complexa e origem multifatorial, acometendo indivíduos, em alta prevalência, de idade superior a 40 anos. Combinando fatores genéticos e ambientais, a DM2 pode ser agravada por hábitos alimentares irregulares e inatividade física, além de forte influência de histórico familiar, sedentarismo, obesidade, dislipidemia, hipertensão arterial e síndrome metabólica, dentre outros fatores. A DM2, na maioria das vezes, é assintomática, o que desfavorece o diagnóstico e o tratamento precoces, diminuindo assim a eficiência e evolução terapêutica. No entanto, ao observar a relevância das medidas de estilo de vida, a adoção da prática dos exercícios físicos nas pessoas com DM, principalmente nos idosos, nota-se efetivo controle glicêmico e controle de morbidades (Freitas et al., 2020). A doença e, principalmente, seu mau controle, podem causar o aumento da glicemia e as altas taxas podem levar a complicações no coração, nas artérias, nos olhos, nos rins e nos nervos, e, desse modo, o objetivo deste estudo é evidenciar as principais complicações crônicas decorrentes do diabetes mellitus.

\section{Metodologia}

Trata-se de uma pesquisa descritiva do tipo revisão narrativa da literatura, que buscou responder quais são as evidências sobre as complicações a longo prazo do diabetes mellitus. A pesquisa foi realizada através do acesso online nas bases de dados National Library of Medicine (PubMed MEDLINE), Scientific Electronic Library Online (Scielo), Cochrane Database of Systematic Reviews (CDSR), Google Scholar, Biblioteca Virtual em Saúde (BVS) e EBSCO Information Services, no mês de outubro de 2021. Para a busca das obras foram utilizadas as palavras-chaves presentes nos descritores em Ciências da Saúde (DeCS): em inglês: "diabetes mellitus", "complications", "nephropathy", "retinopathy", "neuropathy", "diabetic foot", "macrovascular", "microvascular" e em português: "diabetes mellitus", "complicações", "nefropatia", "retinopatia", "neuropatia", "pé diabético", "macrovasculares", "microvasculares".

Como critérios de inclusão, foram considerados artigos originais, que abordassem o tema pesquisado e permitissem acesso integral ao conteúdo do estudo, publicados no período de 2004 a 2021, em inglês e português. O critério de exclusão foi imposto naqueles trabalhos que não estavam em inglês e espanhol, que não tinham passado por processo de Peer-View e que não abordassem a temática do estudo. A estratégia de seleção dos artigos seguiu as seguintes etapas: busca nas bases de dados selecionadas; leitura dos títulos de todos os artigos encontrados e exclusão daqueles que não abordavam o assunto; leitura crítica dos resumos dos artigos e leitura na íntegra dos artigos selecionados nas etapas anteriores. Assim, totalizaram-se 30 artigos científicos para a revisão narrativa da literatura, com os descritores apresentados acima, dos últimos dezessete anos e em línguas portuguesa e inglesa.

\section{Resultados e Discussão}

As complicações diabéticas crônicas são classificadas como microvasculares ou macrovasculares, e contribuem para mortalidade e perda de qualidade de vida de pacientes portadores de diabetes mellitus. Sob essa perspectiva, nota-se que a hiperglicemia desempenha um papel crítico na patogênese das complicações microvasculares, como retinopatia diabética, 
nefropatia incipiente e neuropatia, enquanto a aterosclerose contribui para a patogênese das complicações macrovasculares. Por isso, o principal objetivo do manejo clínico dessa doença é a prevenção dessas complicações, servindo como base para a atual recomendação de se manter a hemoglobina glicada (HbA1c) < 7\% (Zoungas et al., 2014).

Ademais, o aumento de glicose intracelular é o principal determinante do dano tecidual causado pelo diabetes, dano este que pode ser reversível quando restaurada a normoglicemia, ou irreversível, mesmo revertida a hiperglicemia, pois se originou de alterações acumulativas em macromoléculas de vida longa. Os mecanismos bioquímicos propostos para explicar anormalidades estruturais e funcionais associadas com a exposição prolongada dos tecidos vasculares à hiperglicemia foram propostos, conforme ilustrado pela Tabela 1. Além disso, hipertensão arterial sistêmica, tabagismo dislipidemia, obesidade, etnia e genética são fatores de risco para o desenvolvimento de complicações associadas ao DM (Nathan et al., 2014).

Tabela 1: Mecanismos bioquímicos propostos para explicar anormalidades estruturais e funcionais associadas à exposição prolongada dos tecidos vasculares à hiperglicemia.

Aumento da produção intracelular de produtos finais de glicação avançada (AGE), que modificam proteínas intracelulares e plasmáticas, modificando as suas funções e modificam também componentes da matriz extracelular alterando a interação desses componentes.

A via dos poliois, gerando um aumento da atividade da aldose-redutase, que consome mais NADPH do que o normal, interferindo na função dele como cofator da regeneração da glutationa reduzida (antioxidante intracelular)

Aumento de fluxo pela via das hexosaminas, gerando modulação na expressão de proteínas que, em linhas gerais, causariam oclusão capilar e oclusão vascular.

Ativação da proteína quinase $\mathrm{C}$ (PKC) via acúmulo hiperglicemia-induzido de diacilglicerol. Tanto as alterações causadas pelo aumento do fluxo na via do sorbitol, como o aumento da formação de espécies reativas de oxigênio (ROS) hiperglicemiainduzido, podem ser responsáveis por anormalidades no fluxo sanguíneo e todas as outras alterações bioquímicas.

Fonte: Adaptado de Nathan et al. (2014).

\section{- Resistência à insulina e disfunção endotelial}

A manutenção da glicemia normal depende da capacidade funcional das células pancreáticas de secretarem insulina e da capacidade tecidual de responder a esse hormônio, sendo que ela atua no endotélio vascular regulando o crescimento celular, a expressão de genes, a síntese proteica, a redução do influxo de cálcio e possui ação vasodilatadora, relacionada com a estimulação da produção de óxido nítrico (NO) pelo endotélio, sintetizado pelas células endoteliais, por macrófagos e por neurônios (Faselis et al., 2020).

A resistência à insulina (RI) ocorre quando existe uma diminuição da responsividade das células-alvo a níveis normais de insulina circulante, que, por sua vez, ocasiona uma liberação de mais insulina (geração de hiperinsulinemia), na tentativa de se obter uma resposta fisiológica adequada. Assim, a RI contribui para a disfunção endotelial por promover o desenvolvimento da aterosclerose, gerando inflamação, trombose, rigidez das paredes arteriais, e redução na regulação do tônus e fluxo arteriais. No entanto, a RI precede em muitos anos o diagnóstico de doenças como hipertensão, doença arterial coronariana, DM, obesidade e dislipidemia, podendo ser detectada antes de qualquer grau de intolerância à glicose ser evidente (Katsiki et al., 2019).

Aliado a isso, como dito anteriormente, a insulina, em concentrações fisiológicas exerce funções benignas, apresentando efeitos antiaterogênicos, mediados pelo NO, porém, em condições de hiperinsulinemia, o mesmo hormônio passa a estimular a produção de lipídios, a proliferação da célula muscular lisa, a síntese de colágeno e a produção de fatores de crescimento. Concomitantemente, a produção de NO mediada pela insulina se torna comprometida nessas situações (Avogaro et al., 2019). 
O endotélio vascular, formado por células com alta atividade metabólica, quando íntegro, forma uma rede de proteção que permite melhor fluidez sanguínea e evita a coagulação do sangue, sendo considerado um órgão endócrino, que, em resposta a diversos estímulos, sintetiza e libera diversas substâncias vasoativas de ação sinérgica, antagônica ou complementar, exercendo assim, papel fundamental na homeostase vascular. Diversas funções têm sido atribuídas ao endotélio, como a regulação do tônus vascular, através de fatores de relaxamento (NO, prostaciclina, e fator hiperpolarizante do endotélio) e vasoconstricção (endotelina-1, tromboxano A2, angiotensina II e ânion superóxido); ativação e inibição plaquetária, promovidos por fatores endoteliais que promovem vasoconstricção e vasodilatação respectivamente; adesão de leucócitos ao endotélio, promovida pelos fatores vasodilatadores; interação com metabolismo das lipoproteínas; regulação do crescimento vascular; resposta imune inflamatória (Bekele et al., 2019). Assim, quando eventos fisiopatológicos resultam em efeitos deletérios sobre a funcionalidade e integridade do endotélio, ele se torna incapaz de exercer todas essas funções, instalando-se uma disfunção endotelial, que é considerada uma via final comum a diversas doenças cardiovasculares, representando a lesão inicial da aterosclerose evidente (Katsiki et al., 2019)..

\section{- Nefropatia diabética}

A nefropatia diabética (ND) representa, no Brasil, a segunda causa de doença renal crônica (DRC), estando atrás apenas da nefropatia hipertensiva, sendo uma complicação crônica do DM, de longa duração e caráter progressivo, podendo evoluir para insuficiência renal terminal (Maciel et al., 2019). Sob essa perspectiva, nos últimos anos, nota-se a progressão da doença à hiperfiltração e à hipertrofia renal (anormalidades hemodinâmicas e metabólicas das fases iniciais. surgimento de microalbuminúria (excreção de 30 a 300mg/dia) e, frequentemente, após 4 a 5 anos do surgimento da microalbuminúria, se instala a nefropatia diabética clínica, em que a albuminúria excede $300 \mathrm{mg} / \mathrm{dia}$, de forma persistente, e existe um aumento da pressão arterial, com diminuição da taxa de filtração glomerular (TFG) (Ohkuma et al., 2016).

A patogênese da nefropatia diabética é multifatorial, onde estão relacionados fatores hemodinâmicos, metabólicos e inflamatórios (Gross et al., 2005), e dentre os fatores hemodinâmicos, destaca-se a presença de um meio hipermetabólico e de hiperglicemia moderada e de longa duração está associada à vasodilatação da arteríola aferente mais do que na eferente, gerando uma hiperfiltração glomerular, com aumento do fluxo glomerular e elevada pressão intracapilar. Esses fatores ocasionam um estresse hemodinâmico glomerular, precursor da nefropatia diabética (Prabhakar et al., 2007; Thomson et al., 2004).

Ademais, dentre os fatores metabólicos, existem dois de extrema importância, como a hiperglicemia e a glicação de macromoléculas. A glicação de proteínas, aminoácidos, lipídios e ácidos nucleicos ocorre naturalmente e de maneira reversível no organismo (van Buren et al., 2011). Porém, em estados de hiperglicemia, essa glicação se dá de modo irreversível, produzindo os produtos avançados de glicação (AGE), que podem formar ligação cruzada com proteínas de matriz extracelular, da membrana basal e com os receptores dos AGE, levando à endocitose e ativação de mecanismos pró-oxidantes e pró-inflamatórios. O fator de crescimento do endotélio vascular, a nível renal, estimula a produção de NO, gerando vasodilatação e causa lesão vascular por aumento na permeabilidade da barreira de filtração glomerular. Além de estimular a síntese de colágeno IV pelos podócitos, o que gera espessamento na membrana basal (Thomson et al., 2012).

\section{- Neuropatia diabética}

Existem dois grandes grupos de neuropatias diabéticas: as generalizadas/simétricas e as focais/multifocais/assimétricas, em que as diversas formas de manifestações clínicas são agrupadas. Todas são essencialmente sensitivas e pode haver sintomas positivos, como dor e disestesias, que são referidos, muitas vezes, como sensação de queimação, ardência ou choques; e sintomas negativos, como diminuição ou abolição da percepção da dor e temperatura 
(Tesfaye, 2009), e a distribuição desses sintomas corresponde ao segmento do sistema nervoso periférico envolvido. A mais frequente distribuição fenotípica da polineuropatia sensitiva, que ocorre tanto no DM quando no pré-diabetes e em outras doenças metabólicas, é marcada por uma distribuição distal nos membros, predominando os inferiores, com padrão típico de lesão axonal comprimentodependente, conhecido como "em botas" e "em luvas" (Yagihashi et al., 2011). O exame neurológico desses pacientes geralmente revela hipoestesia termoalgésica distal, porém alguns pacientes podem cursar com hipersalgesia e/ou alodinia (Callaghan et al., 2012).

Já a patogênese da polineuropatia e da neuropatia autonômica simétrica distal envolve anormalidades microvasculares e metabólicas, com relação causal entre a hiperglicemia e a progressão da doença. Os sintomas dolorosos, nos pacientes com neuropatia diabética, inicialmente são de leves a moderados, porém, com o avançar da doença e caso não sejam adequadamente tratados, podem se tornar intensos e debilitantes, que costumam ser piores em decúbito e à noite, interferindo no sono desses pacientes. As neuropatias diabéticas de natureza focal decorrem da oclusão das arteríolas endoneurais, com dano isquêmico ao nervo (entretanto vasos sanguíneos epineurais e perineurais também apresentam alterações sugestivas de vasculite). A maioria dos casos de neuropatias diabéticas agudas focais/multifocais geralmente se resolve, pelo menos em parte, e a dor pode ser resolvida dentro de alguns meses, porém a fraqueza pode persistir por mais de um ano para a recuperação total e alguns pacientes ainda apresentam esses sintomas mesmo com o tratamento (Boulton et al., 2019).

\section{- Pé diabético}

Problemas nos pés no diabetes são comuns e caros, e as pessoas com diabetes representam cerca de metade de todas as internações hospitalares por amputações. Como a maioria das amputações no diabetes é precedida por ulceração do pé, é essencial um entendimento completo das causas e do manejo da ulceração. A incidência anual de úlceras nos pés no diabetes é de aproximadamente $2 \%$ na maioria dos países ocidentais, embora taxas mais altas tenham sido relatadas em certas populações com diabetes. Embora até recentemente se acreditasse que o risco de úlceras nos pés ao longo da vida era geralmente de 15 a $25 \%$, dados recentes sugerem que o número pode chegar a $34 \%$, sendo o famoso médico diabético Elliott P. Joslin quem, tendo observado muitos casos clínicos de doença do pé diabético, observou que "a gangrena diabética não é enviada dos céus, mas sim de origem terrena”. Assim, a ulceração do pé não é uma consequência inevitável de se ter diabetes; em vez disso, as úlceras se desenvolvem como consequência de uma interação entre patologias específicas dos membros inferiores e riscos ambientais (Boulton et al., 2019).

\section{- Retinopatia diabética}

A retinopatia diabética é a principal causa de cegueira em pessoas com idade entre 20 e 74 anos (Chaturvedi et al., 2008). Aproximadamente $12 \%$ dos novos casos de diminuição da acuidade visual a um nível que impeça o exercício de atividades laborais, são causados pela retinopatia diabética. Após 20 anos de doença, mais de $90 \%$ dos pacientes com DM tipo 1 e 60\% daqueles com o tipo 2 apresentarão algum grau de retinopatia (Gupta et al., 2013). Nesses pacientes, a principal causa de baixa da acuidade visual é o edema macular, podendo estar presente desde as fases iniciais da retinopatia até em casos nos quais há doença proliferativa grave, acometendo 30\% dos pacientes com mais de 20 anos de diabetes (Matthews et al., 2004). A forma proliferativa é aquela que se relaciona mais frequentemente com a perda visual grave, devido a eventos oculares potencialmente causadores de cegueira irreversível, como a isquemia retiniana difusa, incluindo a macular e o descolamento tracional de retina (Srivastava et al., 2005).

O controle glicêmico adequado é fundamental para a prevenção e diminuição nas complicações relacionadas ao DM, tendo em vista que o tempo de duração do diabetes e o controle glicêmico são os dois fatores mais importantes relacionados ao desenvolvimento e à gravidade da retinopatia diabética (Hainsworth et al., 2019; Li et al., 2019). Ademais, segundo estudos 
sobre o papel da panfotocoagulação, foram estabelecidos critérios diagnósticos para a retinopatia diabética proliferativa e edema macular, respectivamente. Desse modo, evidencia-se, de acordo com as Tabelas 2 e 3, uma classificação baseada na gravidade para a retinopatia diabética e o edema macular, abrangendo os principais critérios diagnósticos. Se edema macular presente, classifica-se, conforme ilustrado pela Tabela 4:

Tabela 2: Classificação de retinopatia diabética

\begin{tabular}{l|l}
\hline Gravidade da retinopatia diabética & Achados à oftalmoscopia sob dilatação pupilar \\
\hline Sem retinopatia aparente & Sem alterações \\
\hline Retinopatia diabética não proliferativa leve & Apenas microaneurismas \\
\hline Retinopatia diabética não proliferativa moderada & $\begin{array}{l}\text { Achados mais abundantes que na retinopatia não proliferativa leve } \\
\text { e menos abundantes que na retinopatia não grave }\end{array}$ \\
\hline Retinopatia diabética não proliferativa grave & $\begin{array}{l}\text { Presença de um dos seguintes achados: mais de 20 hemorragias } \\
\text { retinianas em cada um dos quatro quadrantes retinianos; } \\
\text { ensalsichamento venoso em dois quadrantes ou } \\
\text { microanormalidades vasculares intrarretinianas em um quadrante }\end{array}$ \\
\hline Retinopatia diabética proliferativa & Presença de neovasos e/ou hemorragia vítrea ou pré-retiniana \\
\hline
\end{tabular}

Fonte: Hainsworth et al. (2019).

Tabela 3: Classificação de edema macular diabético

\begin{tabular}{l|l}
\hline Gravidade do edema macular diabético & Achados à oftalmoscopia sob dilatação pupilar \\
\hline Edema macular aparentemente ausente & $\begin{array}{l}\text { Ausência de espessamento retiniano ou exsudatos duros no polo } \\
\text { posterior }\end{array}$ \\
\hline Edema macular aparentemente presente & $\begin{array}{l}\text { Presença de espessamento retiniano ou exsudatos duros no polo } \\
\text { posterior }\end{array}$ \\
\hline
\end{tabular}

Fonte: Hainsworth et al. (2019).

Tabela 4: Classificação de edema macular presente

\begin{tabular}{l|l|l}
\hline Edema macular leve & Edema macular moderado & Edema macular grave \\
\hline $\begin{array}{l}\text { Algum grau de espessamento de retina } \\
\text { ou exsudatos duros no polo posterior, } \\
\text { no entanto, distantes do centro foveal }\end{array}$ & $\begin{array}{l}\text { Espessamento de retina próximo ao } \\
\text { centro da mácula, mas ainda não } \\
\text { atingindo seu centro }\end{array}$ & $\begin{array}{l}\text { Espessamento de retina ou exsudatos } \\
\text { duros, atingindo o centro da mácula }\end{array}$ \\
\hline
\end{tabular}

Fonte: Hainsworth et al. (2019).

O tratamento padrão ouro para o edema macular e retinopatia proliferativa é a fotocoagulação, que impede a perda de visão em $90 \%$ dos casos, quando iniciada nas fases não proliferativa avançada ou proliferativa inicial. Já em pacientes com retinopatia proliferativa de alto risco, a perda de visão grave (20/800 ou pior) é reduzida em $50 \%$ dos casos. Além disso, o tratamento do edema macular clinicamente significativo com laser em grid ou focal/direto reduz o risco de baixa de visão em $50 \%$. Ademais, diversos tratamentos farmacológicos foram propostos para pacientes nessa categoria, como drogas antiangiogênicas (bevacizumabe, ranibizumabe e aflibercepte) e corticoesteroides (triancinolona acetonida), injetados diretamente na cavidade vítrea. Casos em que a fotocoagulação não é eficaz, como nas trações vitreomaculares, hemorragias vítreas persistentes, descolamentos tracionais de retina acometendo a região macular, devem ser tratados cirurgicamente pela vitrectomia. Considerando todos os casos cirúrgicos, a vitrectomia proporciona acuidade visual melhor que 20/100 em cerca de $80 \%$ dos casos (Li et al., 2019).

\section{- Doença cardiovascular}

Desde meados de 1990, notou-se que a presença de DM conferia risco de evento coronariano isquêmico similar ao de indivíduos não-diabéticos sabidamente coronariopatas. Por isso, o DM2 foi chamado de "equivalente coronariano", a partir da 
constatação de que indivíduos sem DM2, mas com infarto prévio, teriam a mesma incidência de infarto agudo do miocárdio (IAM) em 7 anos (de 20\%), do que aqueles com DM2, mas sem histórico de IAM prévio. Aliado a isso, a doença cardiovascular aterosclerótica constitui a principal causa de morte em diversas populações e indivíduos diabéticos apresentam o dobro do risco de morrer por essas causas quando comparados à população geral. Esses pacientes, quando sofrem evento coronariano, têm maior risco de morte que aqueles sem a doença (Yamazaki et al., 2018).

Outrossim, a presença de DM também eleva em 3 vezes a mortalidade por AVC. Além disso, o DM2 geralmente não vem sozinho, haja vista que, geralmente, o paciente apresenta outras anormalidades, como a obesidade visceral, a hipertensão arterial sistêmica (HAS) e a dislipidemia, que contribuem significativamente para aumentar a mortalidade por eventos cardiovasculares. Sob esse cenário, as anormalidades lipídicas são um problema grande em pacientes com DM2, pois costumam apresentar uma dislipidemia caracterizada por hipertrigliceridemia, níveis elevados de apolipoproteína B, modificações qualitativas nas lipoproteínas de baixa densidade (LDL), partículas estas que de modo característico, são pequenas e densas e apresentam baixos níveis de colesterol contido do que na lipoproteína de alta densidade (Ference et al., 2016).

Além disso, o estado pró-trombótico no diabetes é marcado pela agregação plaquetária e por alterações na fibrinólise no ateroma dentro da parede do vaso, pois a hiperglicemia desencadeia: ativação da PKC redutase; redução na produção de óxido nítrico e prostaciclinas; formação de íons oxidantes; desequilíbrio na homeostase do cálcio; formação do tromboxane A2 e aumento na produção de tombina e Fator de von Willebrand. Ademais, a quebra enzimática das partículas de fibrinogênio pode estar inibida em pacientes com DM, predispondo à aterosclerose acelerada, trombose e oclusão arterial aguda. Além disso, os ateromas em pacientes portadores de DM podem exibir uma expresão aumentada de fator tecidual, um potente prócoagulante (Brown et al., 2013).

Outrossim, no diabetes há uma produção aumentada de moléculas de adesão e aumento na sinalização inflamatória, de forma que monócitos e linfócitos T ligam-se mais facilmente à parede da célula endotelial, formando placas ateroscleróticas com composição pró-inflamatória ou hiperinflamatória. Além disso, macrófagos, células que secretam grandes quantidades de citocinas pró-inflamatórias também são mais abundantes nos ateromas de pacientes com DM. A estabilidade da placa também é mantida pela produção de colágeno pelas células musculares lisas, que migram para o local da lesão. Essas células musculares lisas parecem sofrer mais apoptose em pacientes diabéticos e esse evento é atribuído ao desenvolvimento de capilares acelulares mediado pelo sorbinol (Zinman et al., 2015).

Já a hipertensão arterial sistêmica (HAS) está presente em mais de $60 \%$ dos pacientes com diabetes, indepententemente da idade ou da obesidade associada. Os fatores podem estar envolvidos na gênese da HAS, incluem resistência à insulina (pela retensão de sódio e água nos túbulos renais, estimular o sistema nervoso simpático e aumentar a responsividade adrenal à angiotensina), alterações estruturais renais, de estrutura e função vascular, alterações no eixo hipotálamo-hipófise-adrenal, ativação do sistema renina-angiotensina-aldosterona e do sistema nervoso simpático (Yamazaki et al., 2018).

A cardiomiopatia diabética caracteriza-se pela disfunção miocárdica devido hipertrofia miocelular e fibrose miocárdica, e associa-se à redução da função mecânica do coração, anormalidades eletrofisiológicas, defeitos nas organelas celulares e downregulation dos receptores pelos níveis cronicamente elevados de catecolaminas (Parving et al., 2012). Contribuindo com a fisiopatologia da cardiomiopatia diabética, a disfunção endotelial pode levar a episódios repetidos de vasoconstricção e lesão isquemia-reperfusão. Assim, o aumento da permeabilidade vascular associado à disfunção endotelial causa edema intersticial, fibrose e disfunção miocárdica (Imai et al., 2011). 


\section{- Declínio cognitivo e Doença de Alzheimer}

Segundo Corral et al. (2015), um metabolismo inadequado da glicose no cérebro resultante da resistência à insulina, a capacidade reduzida da insulina de estimular a utilização da glicose, está no centro de novos caminhos terapêuticos para tratar a causa mais comum de demência em todo o mundo, ou seja, a doença de Alzheimer, haja vista que é postulado que a essa neuropatologia representa um distúrbio neuroendócrino que se assemelha a uma forma única de DM2, acompanhada de neurodegeneração, que às vezes é considerada diabetes tipo 3 (Menezes et al., 2020). Derivado dessa hipótese, alguns agentes que melhoram a sensibilidade à insulina e que reduzem a hiperinsulinemia têm sido propostos para auxiliar no funcionamento cognitivo de pacientes com DM2 ou doença de Alzheimer.

\section{Conclusão}

Complicações macrovasculares devastadoras (doença cardiovascular) e complicações microvasculares (como doença renal diabética, retinopatia diabética e neuropatia) levam ao aumento da mortalidade, cegueira, insuficiência renal e uma diminuição geral da qualidade de vida em indivíduos com diabetes. Atualmente, o tratamento envolve modificação da dieta, redução de peso, exercícios, medicamentos orais e insulina. Nos últimos anos, avanços importantes foram feitos na patogênese do diabetes que afeta os sistemas cardiovascular, renal e nervoso; visão; e as extremidades inferiores, especialmente os pés. Desse modo, é de suma importância, ao observar a relevância das medidas de estilo de vida, a adoção da prática dos exercícios físicos nas pessoas com DM, principalmente nos idosos, que se efetive o controle glicêmico dos portadores de DM2, e, assim, controlando as morbidades associadas e cronicidades.

\section{Referências}

Avogaro, A., et al. (2019). Complicações microvasculares no diabetes: uma preocupação crescente para os cardiologistas. International Journal of Cardiology, $291(1), 29-35$.

Beleke, B. B. (2019). A prevalência de complicações macro e microvasculares do DM entre pacientes na Etiópia 1990-2017: revisão sistemática. Diabetes e síndrome metabólica: pesquisa clínica e análises, 13 (1), 672-677.

Boulton, A. J. M., et al. (2019). Diagnóstico e tratamento de complicações do pé diabético. American Diabetes Association, 3 (8), 1-16.

Brown, D. M., et al. (2013). Resultados de longo prazo da terapia com ranibizumabe para edema macular diabético: os resultados de 36 meses de dois estudos de fase III: RISE e RIDE. Oftalmologia, 120 (5), 2013-2022.

Callaghan, B. C., et al. (2012). Controle de glicose aprimorado para prevenir e tratar a neuropatia diabética. Cochrane Database Syst Rev., 6 (76), 1-11.

Chaturvedi, N., et al. (2008). Efeito do candesartan na prevenção (DIRECT-Prevent 1) e progressão (DIRECT-Protect 1) da retinopatia no diabetes tipo 1: ensaios clínicos randomizados e controlados por placebo. The Lancet, 372 (7), 1394-1402.

Corral, S. R., et al. (2015). Diabetes and Alzheimer Disease, Two Overlapping Pathologies with the Same Background: Oxidative Stress. Oxidative Medicine and Cellular Longevity, 15 (98), 1-14.

Faselis, C., et al. (2020). Complicações microvasculares do diabetes mellitus tipo 2. Ingenta Connect, 18 (2), $117-124$.

Ference, B. A., et al. (2016). Variação em PCSK9 e HMGCR e risco de doenças cardiovasculares e diabetes. N Engl J Med., 375 (7), $2144-2153$.

Freitas, A. J. S., et al. (2020). Identificação de portadores de diabetes mellitus tipo 2 e incentivo as mudanças no estilo de vida. Journal of Biology \& Pharmacy and Agricultural Management, 16 (3), 9-19.

Gross, J. L., et al. (2005). Nefropatia diabética: diagnóstico, prevenção e tratamento. Diabetes Care, 28 (8), $164-176$.

Gupta, N., et al. (2013). Mansoor S, Sharma A, Sapkal A, Sheth J, Falatoonzadeh P, Kuppermann B, Kenney M. Retinopatia diabética e VEGF. Open Ophthalmol J., 7 (8), 4-10.

Hainsworth, D. P., et al. (2019). Fatores de risco para retinopatia em diabetes tipo 1: o estudo DCCT / EDIC. Diabetes Care, 42 (5), $875-882$.

Hang, X., et al. (2019). Etiology of metabolic syndrome and dietary intervention. International Journal of Molecular Sciences, 20 (128), 1-11.

Imai, E., et al. (2006). Olmesartana reduzindo a incidência de doença renal terminal em ensaio clínico de nefropatia diabética (ORIENT): justificativa e desenho do estudo. Hypertens Res., 29 (7), 703-709. 
Research, Society and Development, v. 10, n. 14, e96101421794, 2021

(CC BY 4.0) | ISSN 2525-3409 | DOI: http://dx.doi.org/10.33448/rsd-v10i14.21794

Katsiki, N., et al. (2019). Obesidade, síndrome metabólica e o risco de complicações microvasculares em pacientes com diabetes mellitus. Ingenta Connect, 25 (18), 2051-2059.

Li, T., et al. (2019). Anormalidades microvasculares retinianas em crianças com diabetes mellitus tipo 1 sem deficiência visual ou retinopatia diabética. Oftalmologia investigativa e ciências visuais, 60 (4), 990-998.

Maciel, R. O., et al. (2019). Nefropatia diabética- incidência e fatores de riscos associados. Braz. J. Hea. Rev; Curitiba, 2 (4), $3808-3823$.

Matthews, D. R., et al. (2004). Riscos de progressão da retinopatia e perda de visão relacionados ao controle rígido da pressão arterial no diabetes mellitus tipo 2: UKPDS 69. Arch Ophthalmol., 122 (7), 1631-1640.

Menezes, L. M. S., et al. (2020). Relação entre doença de alzheimer e diabetes mellitus tipo 2. Brazilian Journal of Health Review, 3 (6), $16326-16334$.

Nathan, D. M., et al. (2014). O controle do diabetes e estudo de complicações / epidemiologia das intervenções do diabetes e estudo de complicações aos 30 anos: visão geral. Diabetes Care, 37 (1), 9-16.

Ohkuma, T., et al. (2016). Efeitos do tabagismo e sua cessação na creatinina e cistatina C taxas estimadas de filtração glomerular e albuminúria em pacientes do sexo masculino com diabetes mellitus tipo 2: o Registro de Diabetes de Fukuoka. Hypertens Res., 39 (7), $744-751$.

Parving, H. H., et al. (2012). Desfechos cardiorrenais em um estudo com aliscireno para diabetes tipo 2. N Engl J Med., 367 (1), $2204-2213$.

Prabhakar, S., et al. (2007). A nefropatia diabética está associada ao estresse oxidativo e à diminuição da produção de óxido nítrico renal. J Am Soc Nephrol., 18 (5), 2945-2952.

Prasad, S., et al. (2014). Diabetes mellitus and blood-brain barrier dysfunction: an overview. Aust J Pharm, 2 (125), $125-131$.

Srivastava, B. K. (2005). A hipertensão desempenha um papel na retinopatia diabética? J Assoc Physicians India, 53 (6), 803-8.

Tesfaye, S. (2009). Avanços na gestão da neuropatia periférica diabética. Curr Opin Suporte Palliat Care, 3 (1), $136-143$.

Thomson, S. C., et al. (2004). Função renal no diabetes precoce: a hipótese tubular da filtração glomerular. Am J Physiol Ren Physiol., 286 (7), 8-15.

Thomson, S. C., et al. (2012). Efeitos agudos e crônicos do bloqueio de SGLT2 na função glomerular e tubular no rato diabético precoce. Am J Physiol Regul Integr Comp Physiol., 302 (8), 75-83.

van Buren, P. N., et al. (2011). Hipertensão na nefropatia diabética: epidemiologia, mecanismos e gestão. Adv Chronic Kidney Dis., 18 (2), $28-41$.

Yagihashi, S., et al. (2011). Mecanismo da neuropatia diabética: onde estamos agora e para onde ir? J Diabetes Investig., 2 (6), 18-32.

Yamazaki, D., Hitomi, H. \& Nishiyama, A. (2018). Hipertensão com complicações do diabetes mellitus. Hypertens Res., 41 (7), $147-156$.

Zinman, B., et al. (2015). Empagliflozina, desfechos cardiovasculares e mortalidade no diabetes tipo 2. N Engl J Med., 373 (4), $2117-2128$.

Zoungas, S., et al. (2014). Impacto da idade, idade no diagnóstico e duração do diabetes no risco de complicações macrovasculares e microvasculares e morte no diabetes tipo 2. Diabetologia, 57 (8), 2465-2574. 